\title{
2.3 $\AA$ X-ray Structure of the Heme-Bound GAF Domain of Sensory Histidine Kinase DosT of Mycobacterium tuberculosis
}

\author{
Larissa M. Podust, Alexandra Ioanoviciu, Paul R. Ortiz de Montellano
}

From the Department of Pharmaceutical Chemistry, University of California, San Francisco, CA 94158-2517.

SUPPLEMENTAL FIGURES

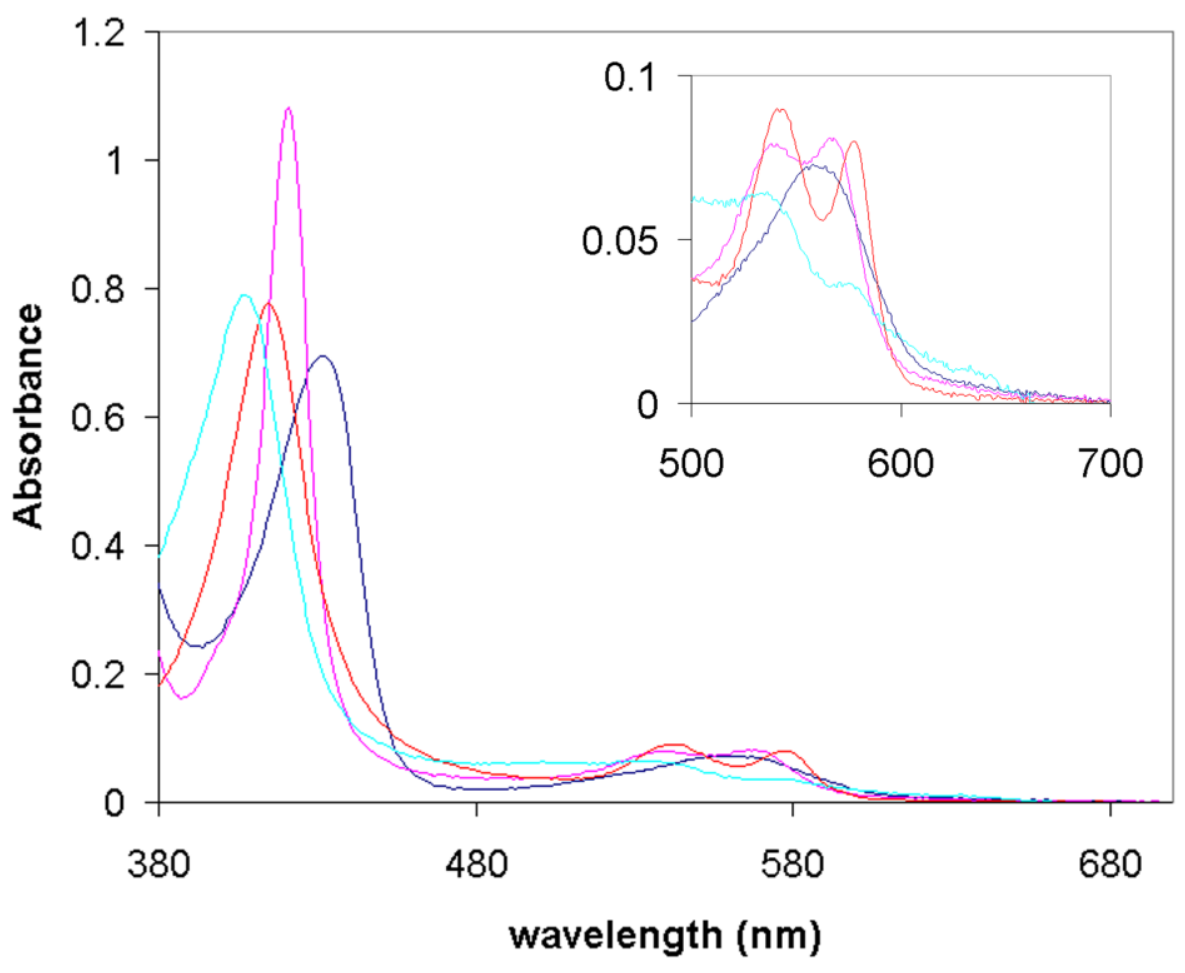

Figure S1. UV-visible spectral characterization of $\mathbf{G A F}_{\text {DosT. }}$ Spectra of the ferric $\mathrm{GAF}_{\text {DosT }}$, cyan; ferrous $\mathrm{GAF}_{\text {DosT }}$, dark blue; ferrous $\mathrm{GAF}_{\mathrm{Dos}} \mathrm{T}^{-} \mathrm{CO}$ complex, magenta; and ferrous $\mathrm{GAF}_{\mathrm{Dos} T}-\mathrm{O}_{2}$ complex, red, are shown. The insert shows an enlargement of the visible region of the spectra. 


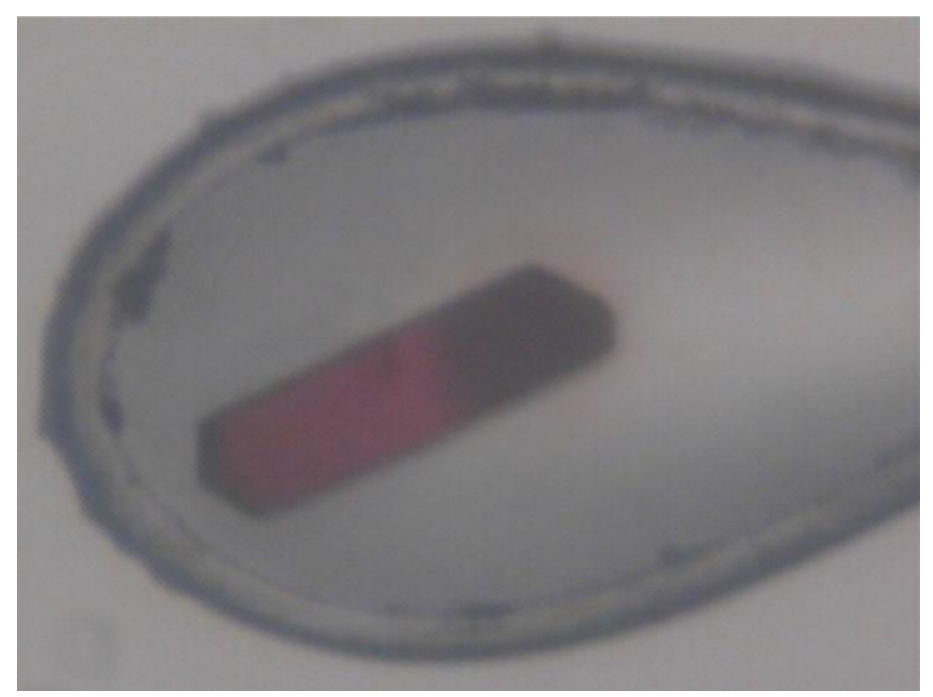

Figure S2. GAF $_{\text {DosT }} \mathbf{x}$-ray induced photoreduction. A snapshot of the $\mathrm{GAF}_{\mathrm{Dos} T}$ crystal after collection of the two-wavelength anomalous diffraction data set is shown. The irradiated part of the crystal on the right is of a much darker red color. 
Fig. S3 (page1)

DosT M.tuberculosis (053473)

DosT_M.tuberculosis (053473) M. smegmatis (ABK70610) M. vanegmatis (ABK70610) M. vanbaalenii (ABM1
M. gilvum (ABP 46316) M. gilvum (ABP 46316)
DosS M.ulcerans (ABL04774) DosS_M.ulcerans (ABL04774) DosS_M.tuberculosis (NP217648) N. farcinica (BAD57741) Rhodococcus_RHA1 (ABG91885) T. fusca (AAZ57079) S. coelicolor (CAB53427) S.tropica (ABP54044) Nocardioides_JS614 (ABL80469)

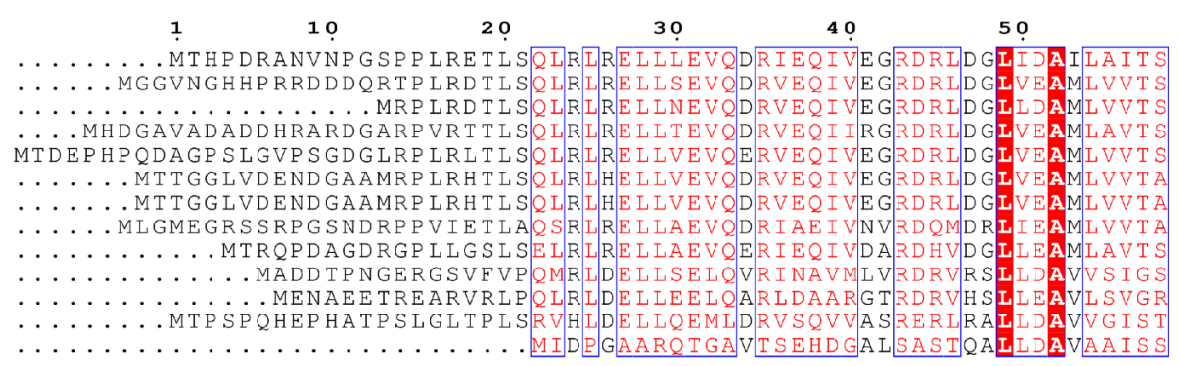

DOsT_M.tuberculosis(053473)

DosT_M.tuberculosis (053473) M. smegmat is (ABK70610)

M. vanbaalenii (ABM12229) M. gi lvum (ABP46316)

MosS M. ulcerans (ABL04774)

DosS_M.ulcerans (ABL04774)

DosS_M.tuberculosis (NP217648)

N. farcinica (BAD57741)

Rhodococcus_RHAl (ABG91885)

T. fusca (AAZ57079)

S. coelicolor (CAB53427)

s. tropica (ABP54044)

Nocardioides_JS614 (ABL80469)

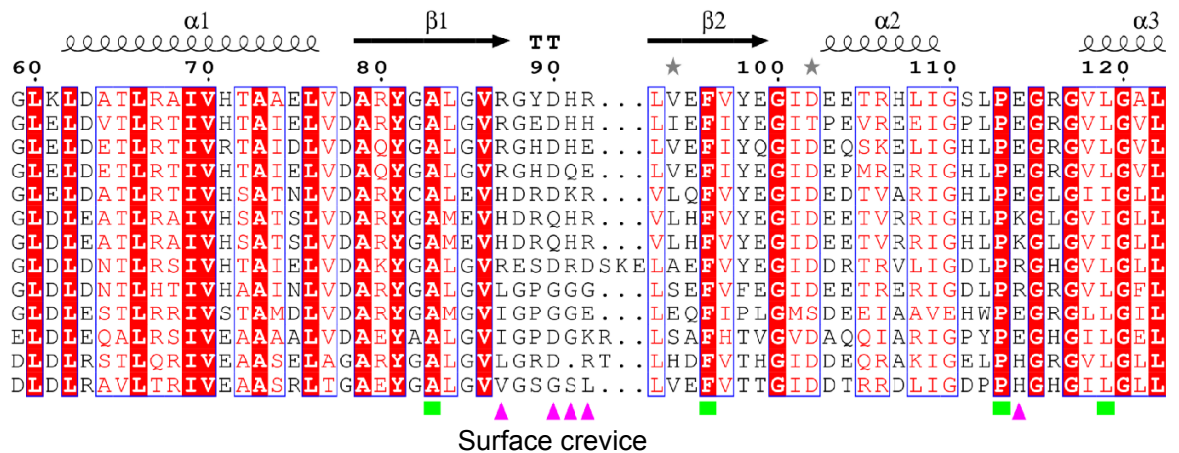

DosT_M.tuberculosis(053473)

DosT_M.tuberculosis (053473)

M. smegmat is (ABK70610)

M. vanbaalenii (ABM12229)

M. gilvum (ABP 46316)

DosS M.ulcerans (ABL04774)

DosS M.bovis (BCG CAL73144)

DosS_M.tuberculosis (NP217648)

N. farcinica (BAD57741)

Rhodococcus_RHA1 (ABG91885)

T. fusca (AAZ $\overline{5} 7079$ )

S. coelicolor (CAB53427)

S.tropica (ABP54044)

Nocardioides_JS614 (ABL80469)

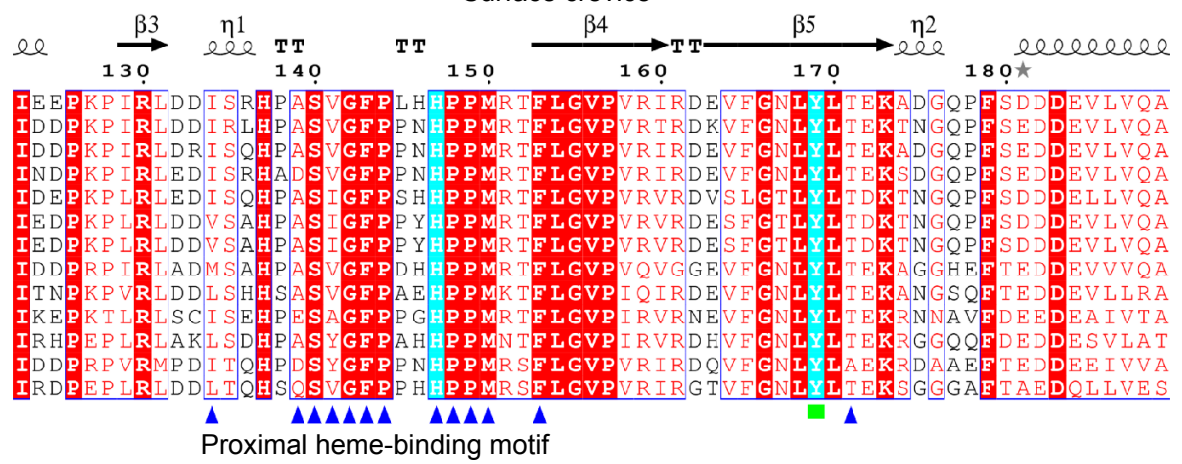

DosT_M.tuberculosis(053473)
DosT_M.tuberculosis (053473)
M.smegmatis (ABK70610)
M.vanbaalenii (ABM12229)
M.gilvum (ABP46316)
DosS_M. ulcerans (ABI04774)
DosS_M.bovis (BCG_CAL73144)
DosS_M.tuberculosis (NP217648)
N.farcinica (BAD57741)
Rhodococcus_RHA1 (ABG91885)
T.fusca(AAZ57079)
S.coelicolor(CAB53427)
S.tropica(ABP54044)
Nocardioides_JS614 (ABL80469)

1900 T

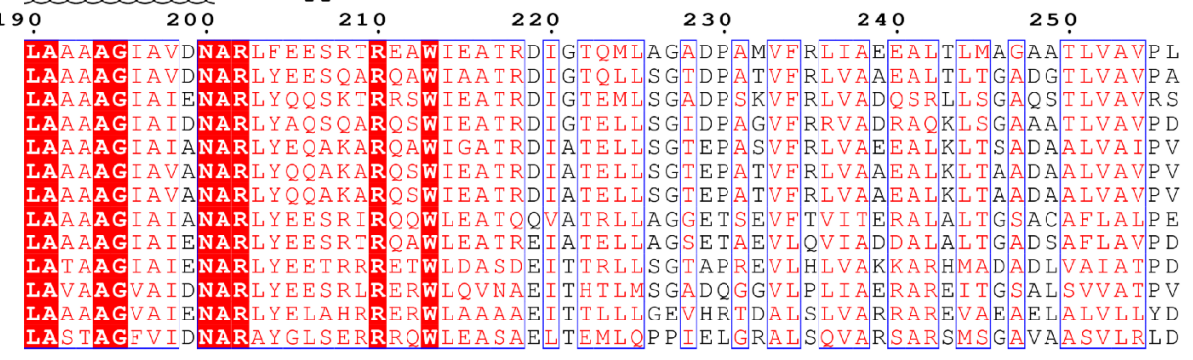

DosT_M.tuberculosis(053473)

DosT_M.tuberculosis (053473)

M. smegmat is (ABK70610)

M. vanbaalenii (ABM12229)

M. gilvum (ABP 46316)

DosS_M.ulcerans (ABL04774)

DosS_M.bovis (BCG_CAL73144)

DosS_M.tuberculosis (NP217648)

N. farcinica (BAD57741)

Rhodococcus RHA1 (ABG91885)

T. fusca (AAZ57079)

S. coelicolor (CAB53427)

S.tropica (ABP54044)

Nocardioides_JS614 (ABL80469)

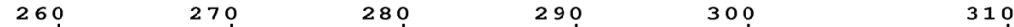

DDEAPACEVDDLVIVEVAGEISPA DPDASAAE . ELVIVEVAGAVPAEVEASAIPVQDNA I GQAFRDRA. . . RRLDV. LD . . . GPGL DLDELDGRVDELVVVATAGD. GPGVSLSAIPTEGSAIGEVFSTKTP....VRFDT. LELE. P. RHLA DP D LPVGEVAELTVAASAGE. TIAAAHDPIPVAGIA I GDAFVHRTP.... GMTDR. ADIG. I. GATV DEDVPASEVAQL LVIEIVGNAVAAAEGCIIAVAG A LAEVLLDSAF . . . RQVDK. I AVEDVDELGD DEDMPAADVGELLVIETVGSAVASTVGRTIPVAGAVLREVFVNGIP... RRVDR. VDLEGLDELAD DEDMPAADVGELIVIETVGSAVAS IVGRTIPVAGAVLREVFVNGIP.... RRVDR. VDLEGLDELAD DP DVP P EDLTELVVVAAAGADADTI TGTRIPMLETH T GVAFRDGRP.... LAVDT. ADAPSEATSLE DPD I P S ....RDEEELVVRIADG. PDASVVRGRSTPVGS TGRAFRGADP I I T E EARESATSP TLLHGLD ....SGIDTLAVELAVG. HEADA WRGIVIPVEGI I GQAFVQRAPVHSADVCRDSRVSAGPPREEG

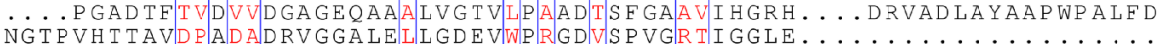


Fig. S3 (page2)

DosT_M.tuberculosis (053473)

DosT_M.tuberculosis (053473)

M. smegmatis (ABK70610)

M. vanbaalenii (ABM12229)

M. gil lvum (ABP 46316)

DosS_M.ulcerans (ABL04774)

Doss M.bovis (BCG CAL 73144)

DosS M.tuberculosis (NP217648)

$N$. farcinica (BAD57741)

Rhodococcus_RHA1 (ABG91885)

T. fusca (AAZ57079)

S. coelicolor (CAB53427)

s. tropica (ABP54044)

Nocardioides JS614(ABL80469)

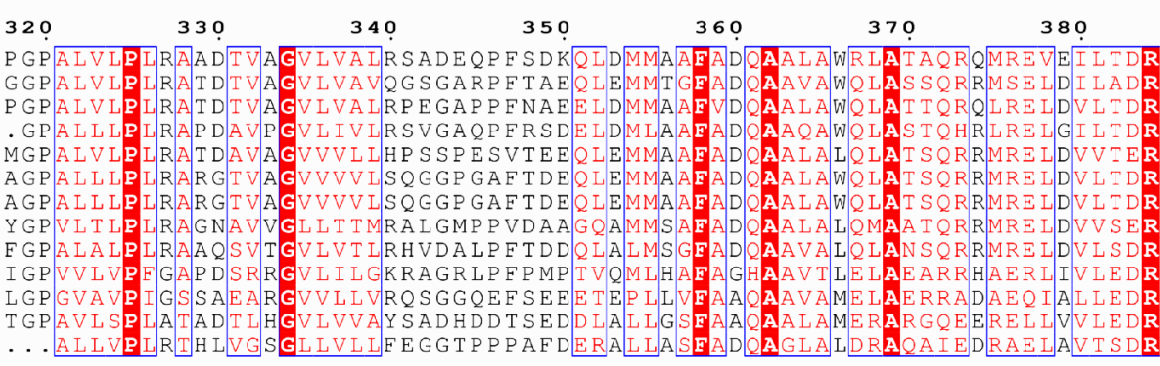

DosT_M.tuberculosis(053473)

DosT_M.tuberculosis (053473)

M. smegmat is (ABK70610)

M. vanbaalenii (ABM12229)

M. gilvum (ABP 46316)

DosS M.ulcerans (ABL04774)

DosS M.bovis (BCG CAI 73144)

DosS_M.tuberculosis (NP217648)

N. farcinica (BAD57741)

Rhodococcus_RHAl (ABG91885)

T. fusca (AAZ57079)

S. coelicolor (CAB53427)

S. tropica (ABF 54044)

Nocardioides_JS614 (ABL80469)

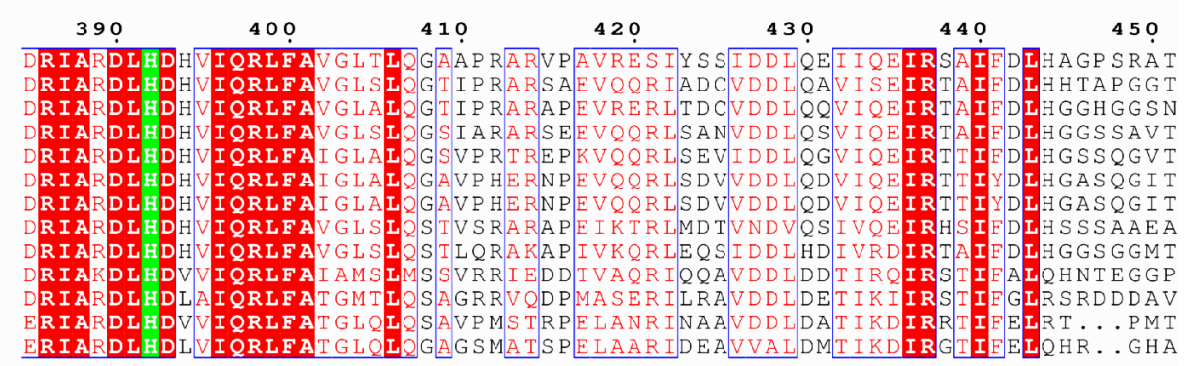

Autophosphorylation site

DosT M.tuberculosis (053473)

DosT_M.tuberculosis (053473)

M. smegmat is (ABK70610)

M. vanbaalenii (AEM12229)

M. gilvum (ABP 4 6316)

DosS M.ulcerans (ABL04774)

DosS M.bovis (BCG CAL 73144)

DosS_M.tuberculosis (NP217648)

N . farcinica (BAD57741)

Rhodococcus_RHAl (ABG91885)

T. fusca (AAZ57079)

S. coelicolor (CAB53427)

S. tropica (ABP54044)

Nocardioides_JS614 (ABL80469)

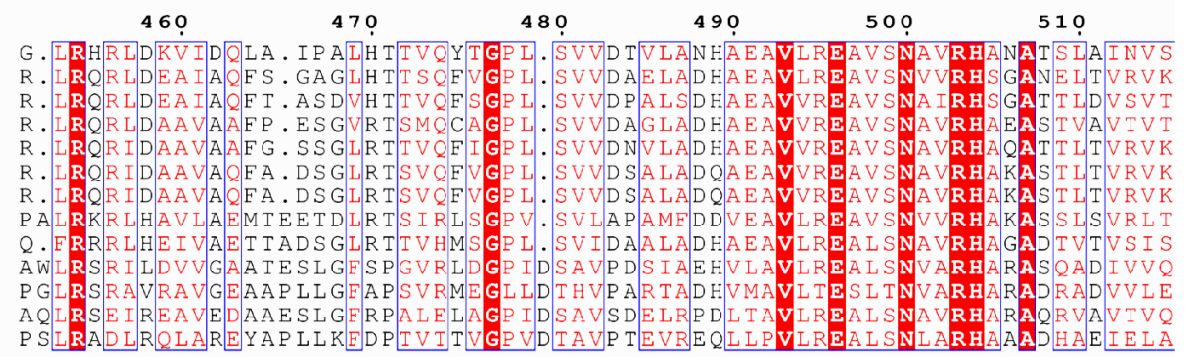

DosT_M.tuberculosis (053473)

DosT_M.tuberculosis (053473)

M. smegmat is (ABK70610)

M. vanbaalenii (ABM12229)

M. gilvum (ABP 46316)

DosS_M.ulcerans (ABL04774)

DOsS M.bovis (BCG CAL 73144)

DosS_M.tuberculosis (NP217648)

N. farcinica (BAD57741)

Rhodococcus_RHAl (ABG91885)

T. fusca (AAZ57079)

S. coelicolor (CAB53427)

S. tropica (ABP54044)

Nocardioides_JS614 (ABL80469)

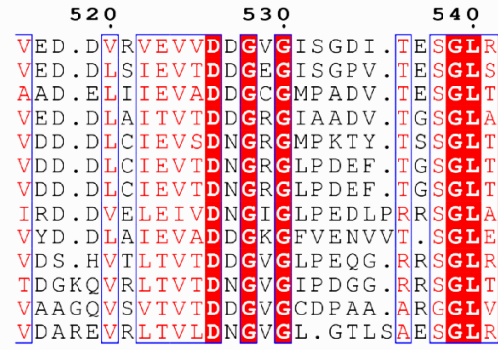

550

560

570

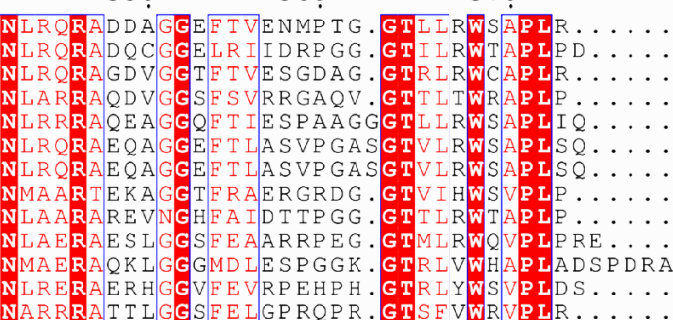

DosT M.tuberculosis(053473)

DosT_M.tuberculosis (053473)

M. smegmat is (ABK70610)

M. vanbaalenii (ABM12229)

M. gilvum (ABP 46316)

DosS_M.ulcerans (ABL04774)

DosS_M.bovis (BCG_CAL 73144)

DosS_M.tuberculosis (NP217648)

N. farcinica (BAD57741)

Rhodococcus RHAl (ABG91885)

T. fusca (AAZ57079)

S. coelicolor (CAB53427)

S.tropica (ABP54044)

Nocardioides_JS614 (ABL80469)

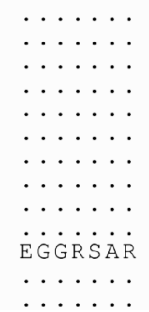


Figure S3. Heme-binding and ligand-binding motifs. Multiple sequence alignments between putative two-component sensory protein-histidine kinases from different organisms are shown. Accession numbers of proteins in Swiss-Prot/TrEMBL (http://us.expasy.org/sprot) or NCBI (http://www.ncbi.nlm.nih.gov) databases are given next to the name of the host organism (M. tuberculosis, Mycobacterium tuberculosis; $M$. smegmatis, Mycobacterium smegmatis; M. vanbaalenii, Mycobacterium vanbaalenii; $M$. gilvum, Mycobacterium gilvum, M. ulcerans, Mycobacterium ulcerans; M. bovis, Mycobacterium bovis; $N$. farcinica, Nocardia farcinica; T. fusca, Thermobifida fusca; $S$. coelicolor, Streptomyces coelicolor, S. tropica, Salinispora tropica). Secondary structure annotation and residue numbering is according to $\mathrm{GAF}_{\mathrm{DosT}}$ of M. tuberculosis. The $\alpha-$ helices are represented by spirals and $\beta$-strands by arrows. Alignments were performed using the MAP algorithm as implemented in the BCM Search Launcher (http://searchlauncher.bcm.tmc.edu/multi-align/multi-align.html). Residues constituting the proximal heme binding motif as deduced from the crystal structure are marked with blue triangles. Residues constituting the distal ligand-binding pocket are marked with green squares. Residues making up the surface crevice are marked with pink triangles. Invariant H147 and Y169 are highlighted in cyan. Highly conserved H392 implicated as the site of autophosphorylation is highlighted in green. The image was prepared by using program ESPript (http://espript.ibcp.fr/ESPript/ESPript/). 